\title{
Molding optical wavefronts: flat optics based on metasurfaces (Presentation Video)
}

Federico Capasso

Federico Capasso, "Molding optical wavefronts: flat optics based on metasurfaces (Presentation Video)," Proc. SPIE 8806, Metamaterials:

Fundamentals and Applications VI, 88060K (17 October 2013); doi: $10.1117 / 12.2030333$

SPIE Event: SPIE NanoScience + Engineering, 2013, San Diego, California, United States 


\title{
Molding Optical Wavefronts: Flat Optics based on Metasurfaces (Presentation Video)
}

\author{
Federico Capasso, Harvard Univ. (United States)
}

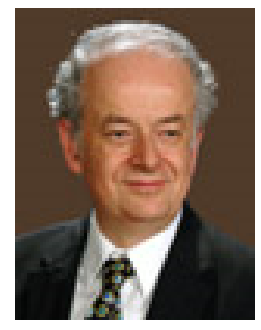

\begin{abstract}
Metasurfaces based on sub-wavelength patterning have major potential for realizing arbitrary control of the wavefront of the diffracted light by achieving local control of the phase amplitude and polarization. We discuss novel devices based on this technique; a salient feature is the ability to create often with a single digital mask an arbitrary analog phase profile. A variety of flat optical components, including lenses, polarizers, vortex plates, coatings, holograms and couplers with polarization invariant coupling efficiency will be presented.
\end{abstract}

View presentation video on SPIE's Digital Library: http://dx.doi.org/10.1117/12.2030333 